City University of New York (CUNY) CUNY Academic Works

2010

\title{
An Application of Statistical Technique to Correct Satellite Data Due to Orbit Degradation
}

Md Zahidur Rahman

CUNY La Guardia Community College

Leonid Roytman

CUNY City College

Run Jesmin

University of London

\section{How does access to this work benefit you? Let us know!}

More information about this work at: https://academicworks.cuny.edu/lg_pubs/51

Discover additional works at: https://academicworks.cuny.edu

This work is made publicly available by the City University of New York (CUNY).

Contact: AcademicWorks@cuny.edu 


\title{
An Application of Statistical Technique to Correct Satellite Data Due to Orbit Degradation
}

\author{
Md Z Rahman ${ }^{1}$, Leonid Roytman ${ }^{2}$, and Runa Jesmin ${ }^{3}$ \\ ${ }^{1}$ Department of Mathematics, LaGuardia Community College of the City University of New York, \\ 31-10 Thomson Avenue, Long Island City, NY-11101 \\ ${ }^{2}$ Department of Electrical Engineering, The City College of the City University of New York, 138 \\ street \& convent Avenue, New York, NY-10031 \\ ${ }^{3}$ Department of Computer Science, King's College London (University of London), Strand London, \\ WC2R2LS, London, UK
}

\begin{abstract}
This paper apply an statistical technique to correct radiometric data measured by Advanced Very High Resolution Radiometers(AVHRR) onboard the National Oceanic and Atmospheric Administration (NOAA) Polar Orbiting Environmental Satellites(POES). This paper study Normalized Difference Vegetation Index (NDVI) stability in the NOAA/NESDIS Global Vegetation Index (GVI) data for the period 1982-2003. AVHRR weekly data for the five NOAA afternoon satellites NOAA-7, NOAA-9, NOAA-11, NOAA-14, and NOAA-16 are used for the China dataset, for it includes a wide variety or different ecosystems represented globally. GVI has found wide use for studying and monitoring land surface, atmosphere, and recently for analyzing climate and environmental changes. Unfortunately the POES AVHRR data, though informative, can not be directly used in climate change studies because of the orbital drift in the NOAA satellites over these satellites' life time. This orbital drift introduces errors in AVHRR data sets for some satellites. To correct this error of satellite data, this paper implements Empirical Distribution Function (EDF) which is a statistical technique to generate error free long-term time-series for GVI data sets. It allows one to represent any global ecosystem from desert to tropical forest and to correct deviations in satellite data due to orbit degradation. The corrected datasets can be used as proxy to study climate change, epidemic analysis, and drought prediction etc.
\end{abstract}

Keywords: Environmental, climate, orbit, satellite, degradation

\section{INTRODUCTION}

Our research investigates the Normalized Difference Vegetation Index (NDVI) data stability calculated from the AVHRR observations for the period 1982-2003 [3]. AVHRR weekly data for the five NOAA afternoon satellites NOAA-7, NOAA-9, NOAA-11, NOAA-14, and NOAA-16 are used for the China dataset. It was found that data for the years 1988, 1992, 1993, 1994, and 2000 are not stable enough compared to other years because of satellite orbit drift, AVHRR sensor degradation, and electronic and mechanical satellite systems deterioration/failure etc. For our research the data for NOAA-7(1982, 1983), NOAA-9 $(1985,1986)$, NOAA-11(1989, 1990), NOAA-14(1996, 1997), and NOAA$16(2001,2002)$ are assumed to be standard due to the fact, that equator crossing time of satellite between 1330 and 1500 , which maximized the value of coefficients[2,8]. In other years, quality of satellite observations significantly deviates from the standard. In this research, we apply Empirical Distribution Function (EDF) which is most effective statistical technique to correct this deficiency of data for the affected years.

For almost two decades, the Advanced Very High Resolution Radiometer (AVHRR) on NOAA polar-orbiting satellites have observed radiances, which were collected, sampled, and stored for the entire world. These data were intensively used by the global community for studying and monitoring land surface, atmosphere, and recently for analyzing climate and environmental changes [1] [2]. AVHRR data, though informative, can not be directly used in climate change studies because of the orbit drift in the NOAA satellites (particularly, NOAA-9, -11, and -14) over these satellites life time [5] [6]. Price 1991 attributed this drift to the selection of a satellite orbit designed to avoid direct sunshine on the instruments. This orbital drift leads to the measurements of Normalized Difference Vegetation Index (NDVI) are being

Earth Resources and Environmental Remote Sensing/GIS Applications, edited by Ulrich Michel, Daniel L. Civco, Proc. of SPIE Vol. 7831, 78310Z · (c) 2010 SPIE · CCC code: 0277-786X/10/\$18 · doi: 10.1117/12.864175 
taken at different local times during the satellites life time, thereby introducing a temporal inconsistency in the NDVI data. Consequently, an orbital drift introduces errors in AVHRR data sets for some satellites.

To correct this error, we consider two statistical techniques: empirical distribution functions, and trend estimation method. Based on our comparison with other method, for the normalization of AVHRR data in this research, EDF method is more accurate than the other method. The main disadvantage of other method is that they correct data for all years. But when using EDF Method, we do not need to correct data for the first two years for each satellite since the first two years produce data of good quality. In addition, EDF is an exact technique for satellite data normalization which depends on an adequate sample size for the approximations to be valid. Therefore, we use the EDF method to correct satellite data in this research because normalized data are relatively closer to the standard data. Moreover, unlike other method, we do not need to correct all year's data. These factors provide strong support to the contention that normalization by EDF is a more efficient method for removing the drift effect and sensor degradation. The main goal of this paper to correct the NDVI and data calculated from the AVHRR observations for the years 1988, 1992, 1993, 1994, 1995, and 2000 by the method of empirical distribution functions (EDF) compared to the standard data. We can use the same methodology globally to create vegetation index to improve the climatology. The corrected datasets can be used as proxy to study climate change, epidemic analysis, and drought prediction etc.

\section{STUDY AREA}

For our research, we wanted to choose an area with diverse ecosystems. China has all major types of ecosystems present in the world. Therefore, we investigate NDVI stability during 1982-2003 over China. It also reduces the amount of data to a manageable state and captures global variety of ecosystems in a single geographic region. It is very difficult to analysis of AVHRR data globally because of the huge volume of data. We can use the same methodology (EDF) globally to create vegetation index to improve the climatology because characteristics of ecosystems are same all over the world based on climate and human activities. For example, we can use the same methodology for Arizona desert in USA and Forest area near Amazon River in South America to correct satellite data due to orbital drift and AVHRR sensor degradation. The land targets approximately of $20_{0} \mathrm{~N}$ to $450 \mathrm{~N}$ in latitude and $720 \mathrm{E}$ to $133_{0} \mathrm{E}$ in longitudes were selected for China

(Fig. 1).

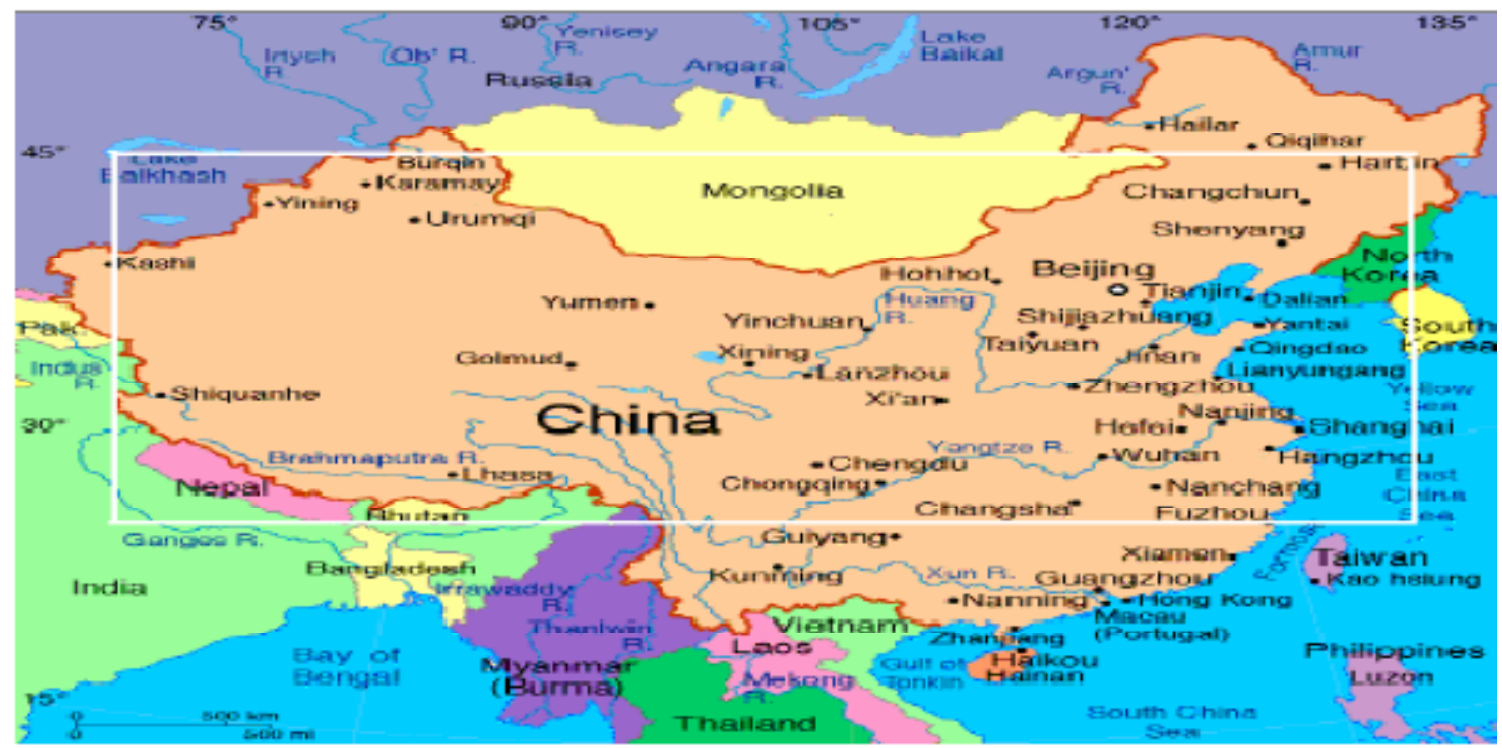

Fig. 1. Geographical Map of China with the area study (bordered area)

We attempted to select relatively small uniform areas using common knowledge of geography, climate, ecosystem, and human activities. The main cover types are desert, forest, and grassland.

\section{DATA AND PROCESSING}

Satellite data were collected from the NOAA/NESDIS Global Vegetation Index (GVI) data set [7] [8] which is one of 
the most widely used satellite products worldwide. The GVI is produced by sampling and mapping the 4-km daily radiance in the VIS $(\mathrm{Ch} 1,0.58-0.68 \mu \mathrm{m})$, NIR $(\mathrm{Ch} 2,0.72-1.1 \mu \mathrm{m})$ in Figure 2 measured onboard NOAA polar-orbiting satellites, to a 16-km map. To minimize cloud effects, these maps, including the NDVI, solar zenith angle, and satellite scan angle, are composited over a 7- day period by saving those values that have the largest difference between VIS and NIR reflectance for each map cell. The weekly GVI data from January 1982 through January 1985 for NOAA-7, from April 1985 through September 1988 for NOAA-9, from October 1988 through August 1994 for NOAA-11, from March 1995 through December 2000 for NOAA-14, and from January 2001 through December 2003 for NOAA-16 were used here.

During 1985-2000, the performance of the channel 1 and 2 differed between NOAA-9, NOAA-11, and NOAA-14 satellites and most importantly, degraded over time for each satellite differently. Since there is no in-flight calibration of channel 1 and 2 of the AVHRR, the question arises as to the validity of the pre-launch calibration coefficients, both in the early days after launch and, perhaps more seriously, after the AVHRR has been in space for a long time. There is a clear evidence in several environmental products, such as the normalized difference vegetation index, global cloud morphology, and earth radiation budget [9], that are generated from channel -1 and channel-2 AVHRR data to indicate that the performance of the instrument these two channels has deteriorated after launch. The need to correct for this inorbit

degradation has been keenly felt recently since it is now being proposed to use the long-term records of AVHRRderived environmental products in climate and global change studies [11] [12] [13] [14] [15] and the degradation of the instrument with time is clearly illustrated by the results shown in Figure 3. Therefore, the standard data preparation procedure for the 7-day composite time series now includes a correction of Ch1 (VIS) and Ch2 (NIR) values following Rao and Chen [13].

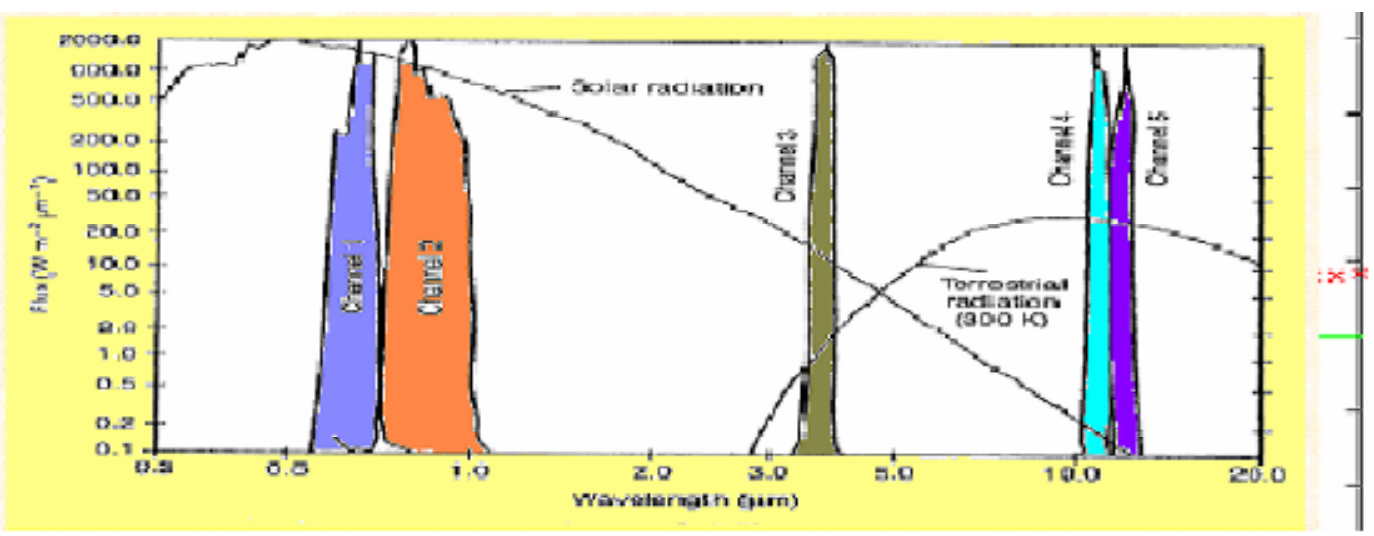

Fig. 2. Normalized spectral response of AVHRR

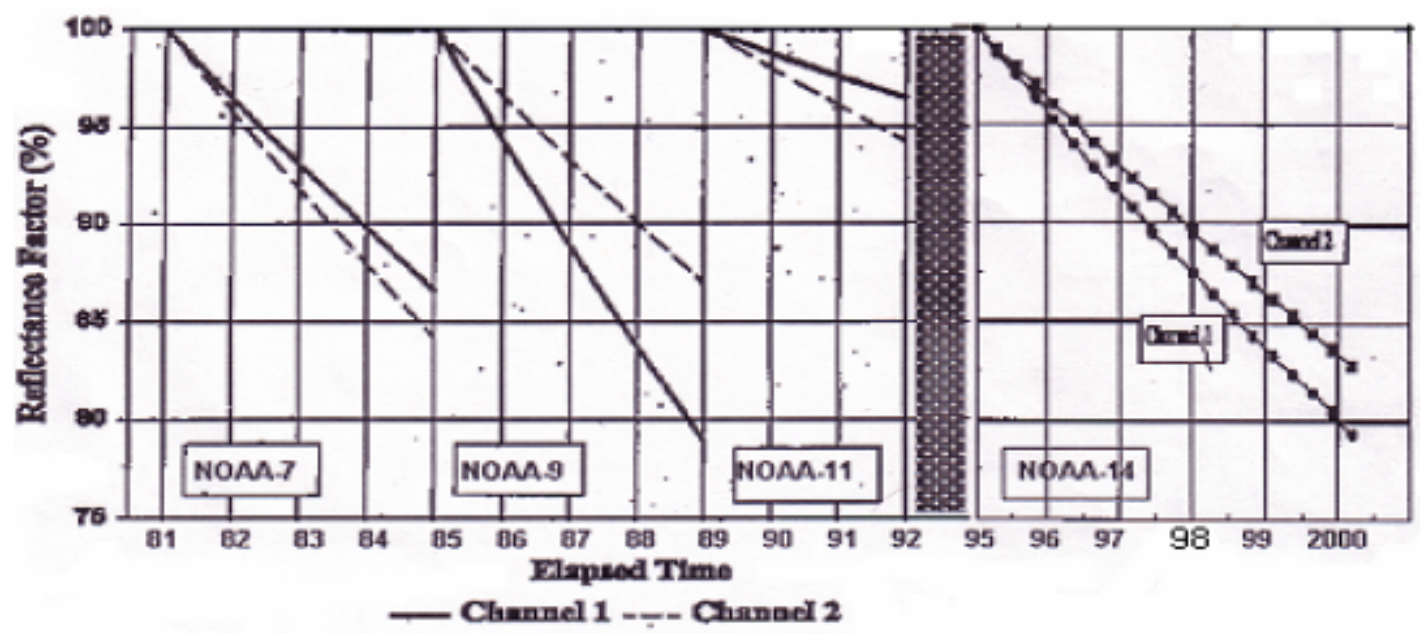

Fig. 3. Degradation of AVHRR Channels 1 and 2 [Rao, 1994] 


\section{METHODOLOGY}

For each satellite, we construct the NDVI time series and also approximate linear trend using least square technique. From trend equation, we estimate two values: the largest difference $((\mathrm{dNt})$ between NDVI at the beginning $(\mathrm{Nb})$ and the end $(\mathrm{Ne})$ of satellite life and difference (dNs) between NDVI at the beginning of the next (n) satellite (Nbn) and at the end

of the previous (p) one (Nep).

$\mathrm{dN}_{\mathrm{t}}=100 *\left(\mathrm{Ne}_{\mathrm{e}}-\mathrm{Nb}\right) /\left(\mathrm{N}_{\mathrm{b}}\right) ; \mathrm{dN}_{\mathrm{s}}=100 *\left(\mathrm{~N}_{\mathrm{bn}}-\mathrm{Nep}_{\mathrm{ep}}\right) / \mathrm{Nep}_{\mathrm{ep}}$

If the dNt values are positive then the NDVI time series upward trend and downward for negative value; positive dNs indicate larger NDVI at the end of the previous satellite and smaller NDVI in the opposite case.

There is no available physical method that can be used to correct for the stability of NDVI. Therefore, we developed a statistical model for the correction of NDVI. The empirical distribution function (EDF) is a statistical technique which is used to generate a normalization data of the years 1988, 1992, 1993, 1994, 1995 and 2000 compared with standard Empirical distribution function (EDF) approach is based on the physical reality, that each ecosystem may be characterized by very specific statistical distribution, independent of the time of observation. It is the best available technique to normalize satellite data. It allows us to represent global ecosystem from desert to tropical forest and to correct extreme distortions in satellite data related to technical problem. To generate the normalization data, we begin by selecting samples of unnormalized earth-scene data covering as much of the range intensities as possible. For NOAA satellites, the area will be rectangular, extending several thousand pixels from desert to tropical forest (both east to west and north to south). Corresponding to the incoming radiance from any pixel, the instrument will respond with an output, $\mathrm{x}$. One can compile the discrete density function, i.e., the histogram, describing the relative frequency of occurrence of each possible count value, for each year. For year $\mathbf{i}$, which is the year to be normalized, let the histogram be Pi(x). An empirical distribution function (EDF) Pi(x) can then be generated; viz.[10],

$$
\left.\mathrm{P}_{\mathrm{i}}^{\mathrm{x}} \mathrm{x}\right)=\Sigma \mathrm{p}_{\mathrm{i}}(\mathrm{t})
$$

The EDF is also known as a cumulative histogram of relative frequency. It is a non-decreasing function of $\mathrm{x}$, and its maximum value is unity.

For convenience, however, we have chosen the maximum value to be 1 ; i.e., if the maximum possible output in counts is $\mathrm{x}$, then $\mathrm{Pi}(\mathrm{x})=1$, as shown in Figure 4

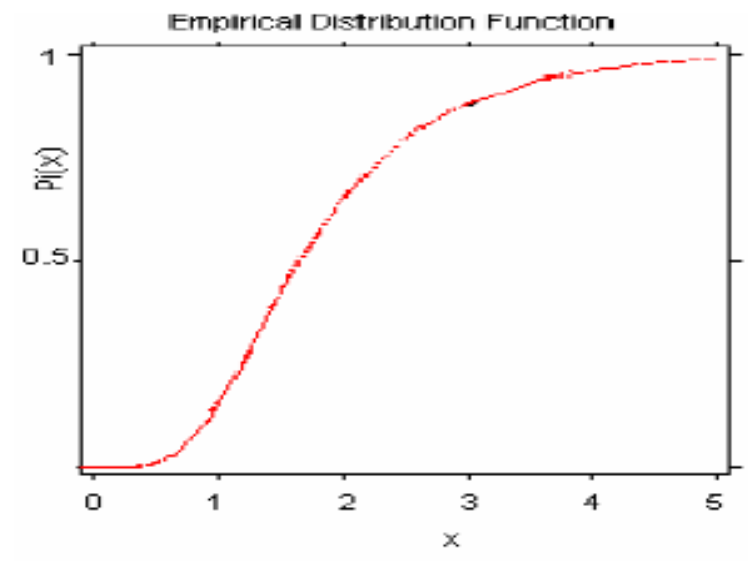

Fig. 4. Empirical Distribution Function In these terms, the basic premise of normalization is that for each output value $\mathrm{x}$ in year $\mathrm{i}$, the normalized value $\mathrm{x}$ ' should satisfy [10]

$$
\mathrm{Ps}_{\mathrm{s}}\left(\mathrm{x}^{\prime}\right)=\mathrm{Pi}(\mathrm{x}),
$$


Where the subscript $\mathbf{s}$ refers to the standard year. In practice, not only is $\mathrm{P}_{\mathrm{s}}$ non-decreasing, but it is also monotonically increasing as a function of $\mathrm{x}^{\prime}$ in the domain of $\mathrm{x}^{\prime}$ where there are data. Therefore, it can be inverted, yielding the solution for $\mathrm{x}^{\prime},[10]$

$$
\mathrm{x}^{\prime}=\mathrm{P}_{\mathrm{s}-1}(\mathrm{Pi}(\mathrm{x}))
$$

When it is applied sequentially for every possible count value $\mathrm{x}$, equation 4 generates the normalization data relating each $\mathrm{x}$ to an $\mathrm{x}$ '. Fig. 5 shows how the procedure is applied in actual practice to generate the normalization data [4] [10]. The figure shows idealized EDF's for the standard and unnormalized years i. In the figure the EDF's are continuous, but in practice they are discrete, being specified only integer values of $\mathrm{x}$.

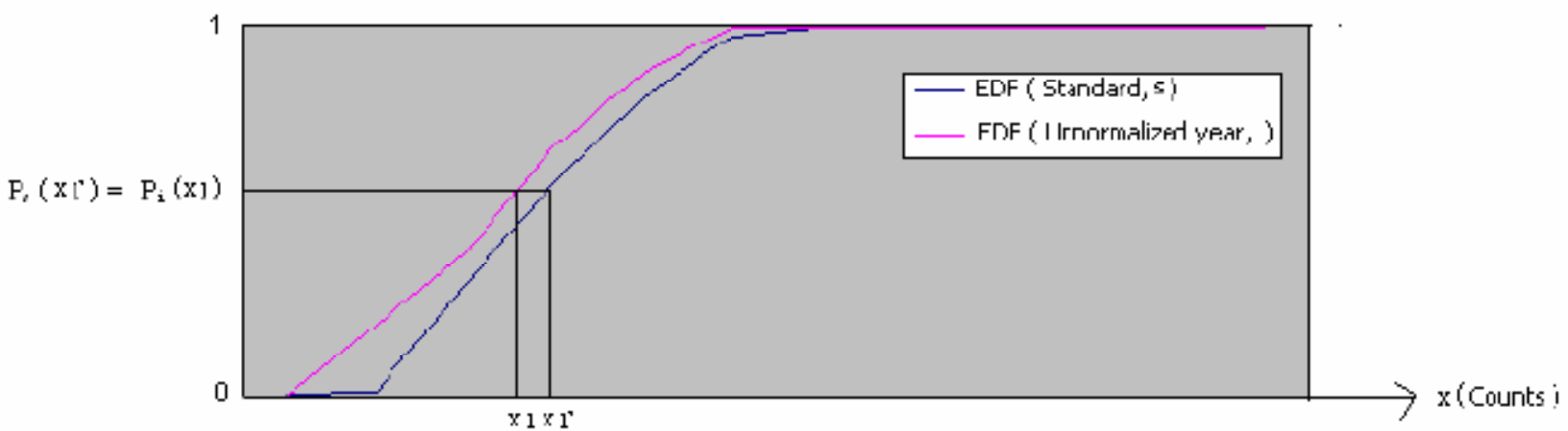

Fig. 5. Example of procedure to generate normalization data

To find $x^{\prime} 1$, the normalized count value corresponding to the unnormalized count value of $\mathrm{x}_{1}$, the following is the procedure: First, for the count value $\mathrm{x} 1$ in unnormalized year $\mathrm{i}$, find the decimal or percentage value from the EDF of year i. In the illustration it is $\mathrm{Pi}(\mathrm{x} 1)$. Then find the point on the standard year's EDF with the same decimal or percentage value. According to equation 3, that decimal or percentage can also be expressed as $\mathrm{P}_{\mathrm{s}}\left(\mathrm{x}^{\prime} 1\right)$. Finally, use the EDF of the standard year to find the normalized count value $\mathrm{x}$ ' 1 . Since the data are actually discrete, we will need to interpolate within the EDF of the standard year to find the value $\mathrm{x}^{\prime}$. Using this technique, we can generate the normalization data. Therefore, we choose EDF method for the normalization of satellite data.

\subsection{COMPARISON OF EDF WITH TREND ESTIMATION METHOD}

We produce NDVI time series for unnormalized data (old NDVI) for NOA-9, correct data (new NDVI) using EDF and trend estimation method, and compared trend estimation method with the EDF method (Figure 6). We notice that the EDF method is better because the Trend Estimation Method corrects all years (Figure 6-a).But when using EDF Method, we do not need to correct the first two years for each satellite since the first two years produce data of good quality. Therefore, we use the EDF method to correct satellite data in this research because normalized data are relatively closer to the standard data. In addition, unlike the trend estimation method, we do not need to correct all year's data.

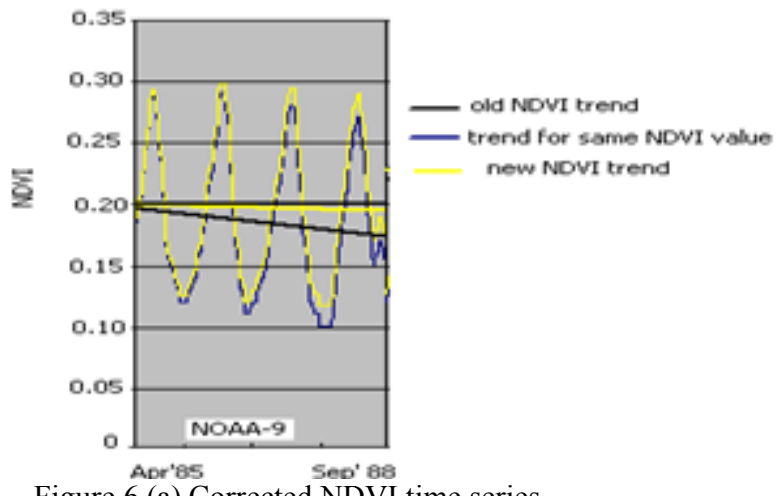

Figure 6 (a) Corrected NDVI time series (yellow line) by the trend estimation method

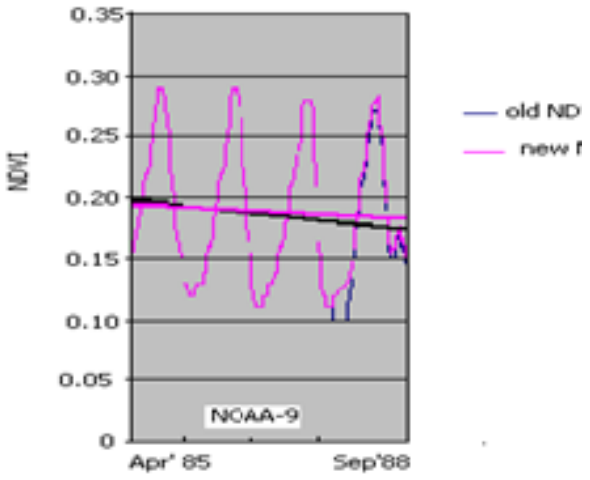

Figure 6 (b) Corrected NDVI time series (pink line) by the EDF method 


\section{RESULTS AND DISCUSSION}

We produce NDVI time series of five NOAA satellites, which is illustrated in Figure 7.

NDVI time series (1962-2003)

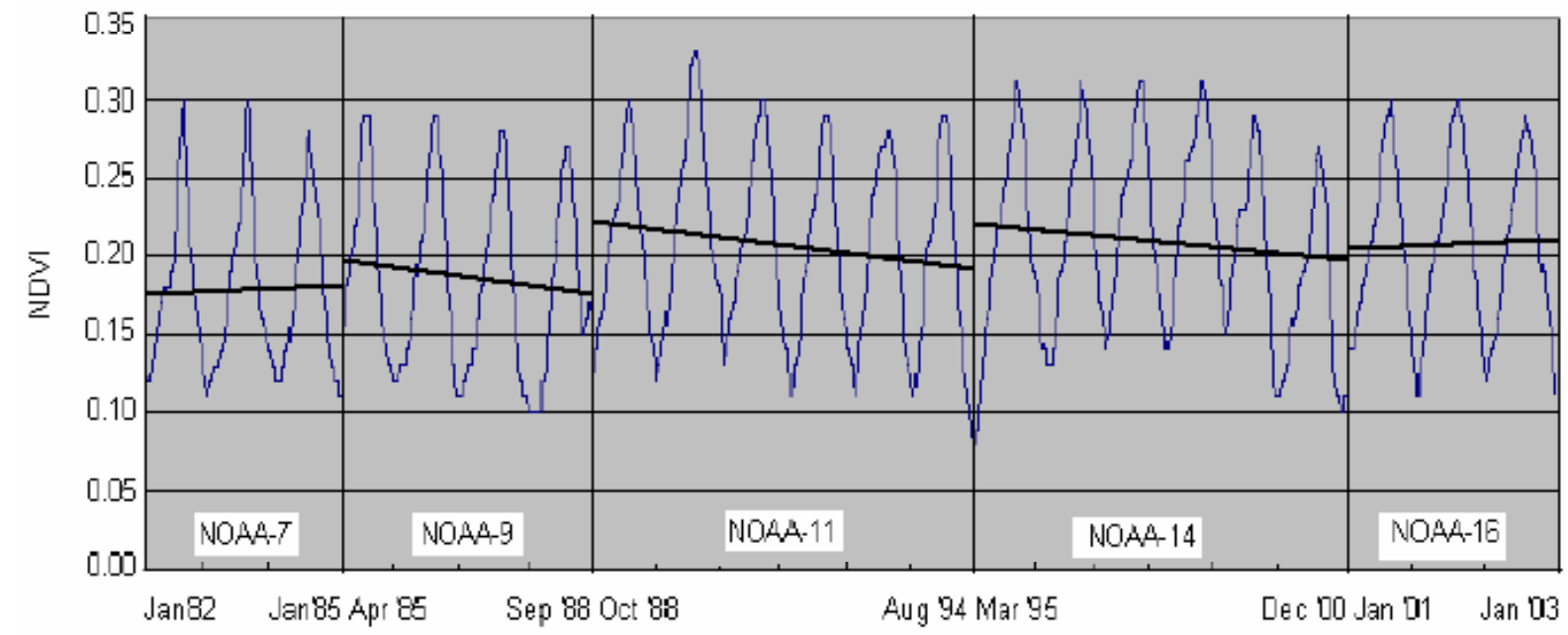

Fig. 7. NDVI time series (yearly old NDVI data) for study area in China.

Data from the afternoon polar orbiters is preferred for producing the NDVI time series because of the high sun elevation angle (low solar zenith angle). However, the equator crossing time drifts to a later hour as the satellites age [6]. Satellite orbit drift results in a systematic change of illumination conditions which is one of the main sources of non-uniformity in multi annual NDVI time series. Figure 7 Shows that the NDVI data of 1988, 1992, 1993, 1994, 1995 and 2000 are nonuniform compared to other years because of satellite orbital drift, and sensor degradation. Therefore, we need to correct the data of those years. We apply EDF for the correction of data of those years. First, EDF construct for unnormalized data and than generate the normalize data compared with standard. Figure 8 shows how the procedure is applied in actual practice to generate normalization NDVI value [4[ [10]. The figure shows idealized EDF's for the standard and the year of 1988. As EDF are based on cumulative histogram, they are discrete. But in Figure 8 they are shown as continuous function.

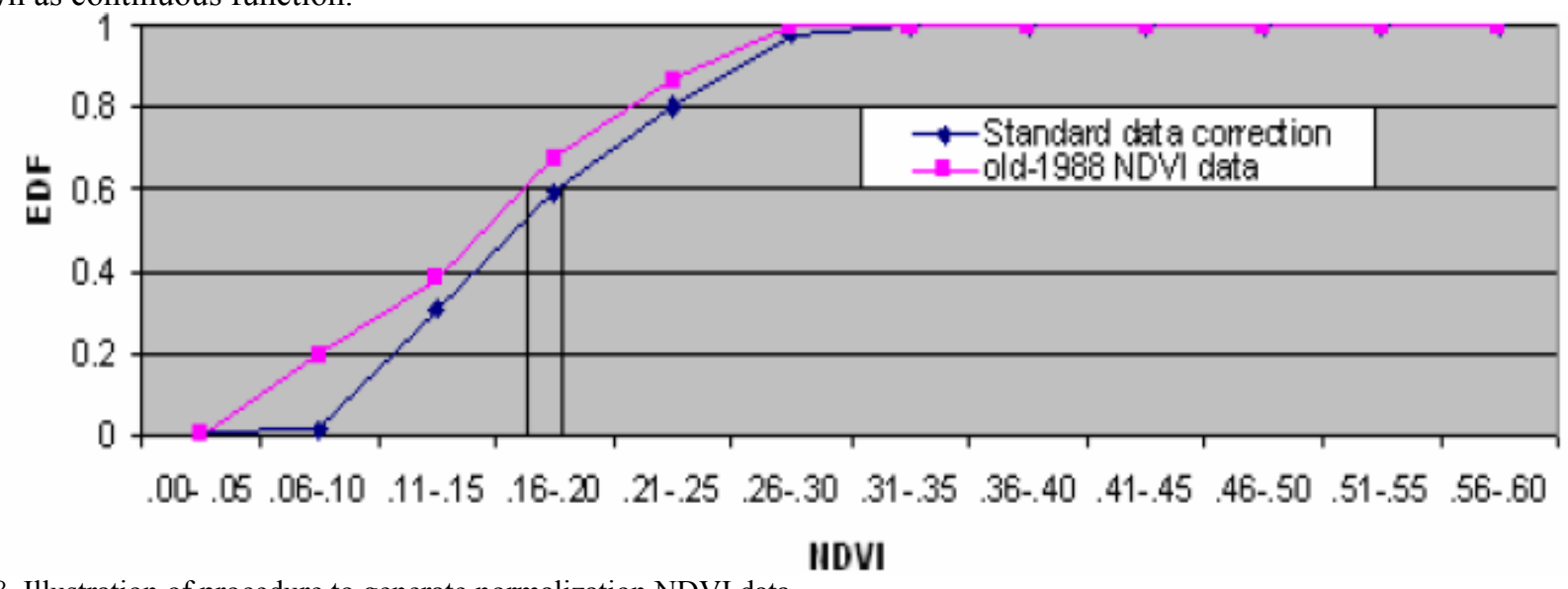

Fig. 8. Illustration of procedure to generate normalization NDVI data.

For example, for the NDVI value 0.16 in year 1988 (Fig. 7) find the value from the EDF of year 1988. In the illustration it the $\mathrm{EDF}_{88}$ is 0.6. Then find the point on the standard year's EDF with the same EDF value. According to equation 3, that the EDF value can also be expressed as the $\mathrm{EDF}_{\text {standard }}$ is 0.6. Finally, use the EDF of the standard year to find the 
normalized count value 0.18 . Since the data are actually discrete, we will need to interpolate within the EDF of the standard year to find the value of 0.18 . Therefore,

New NDVI value for $1988=\mathrm{NDVI}_{1988}+\left(\mathrm{NDVI}_{\text {standard }}-\mathrm{NDVI}{ }_{1988}\right)$ or

New NDVI value for $1988=0.16+(0.18-0.16)=0.18$

Using this technique, EDF's produce to normalize or correct data for the years 1988, 1992, 1993, 1994, 1995, and 2000 compared with standard which are illustrated in following Figures.

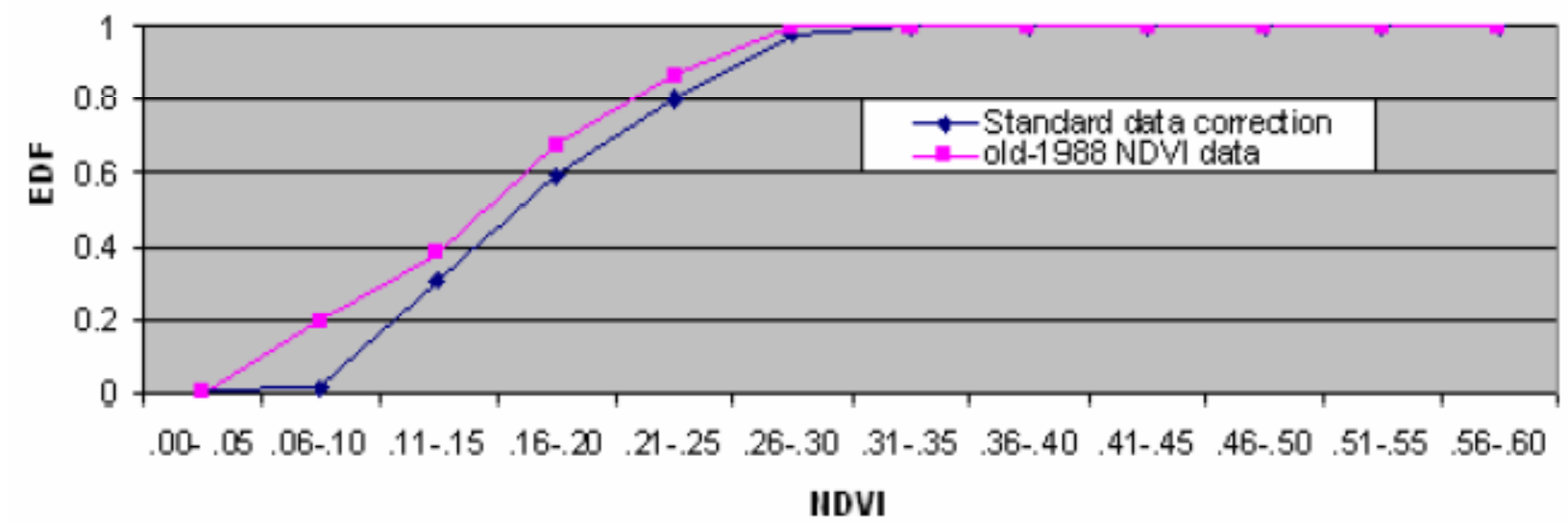

Fig.9. Empirical distribution functions for unnormalized data of 1988 compared with standard data correction sets (subset1)

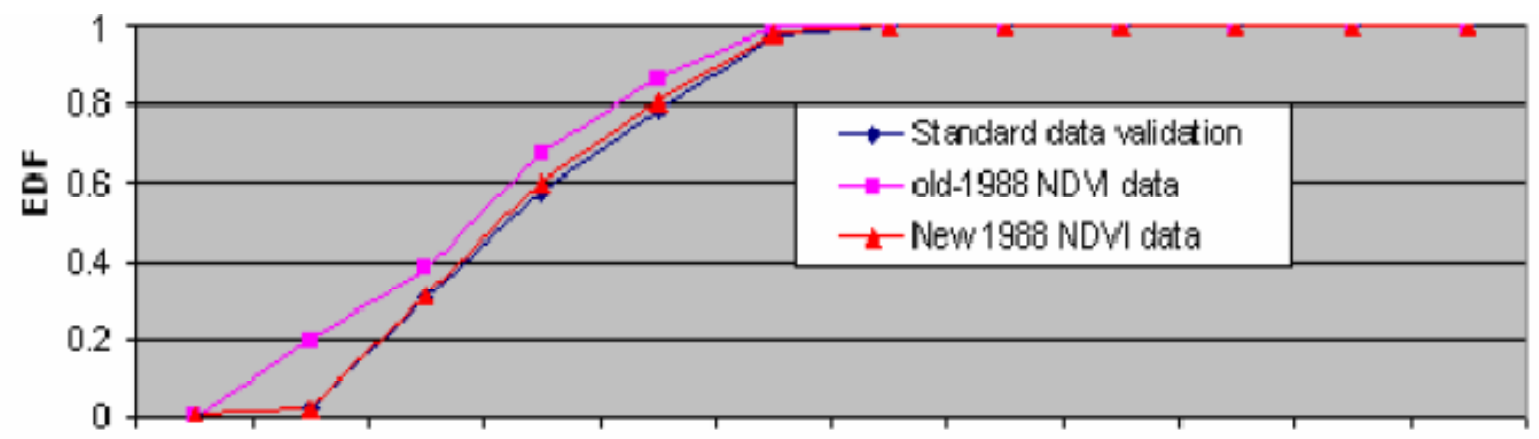

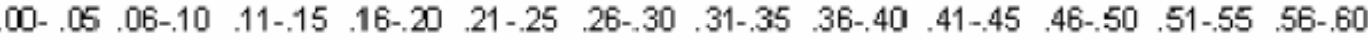

IIDVI

Fig.10. Empirical distribution functions for normalized data of 1988 compared with standard data validation sets (subset 2)

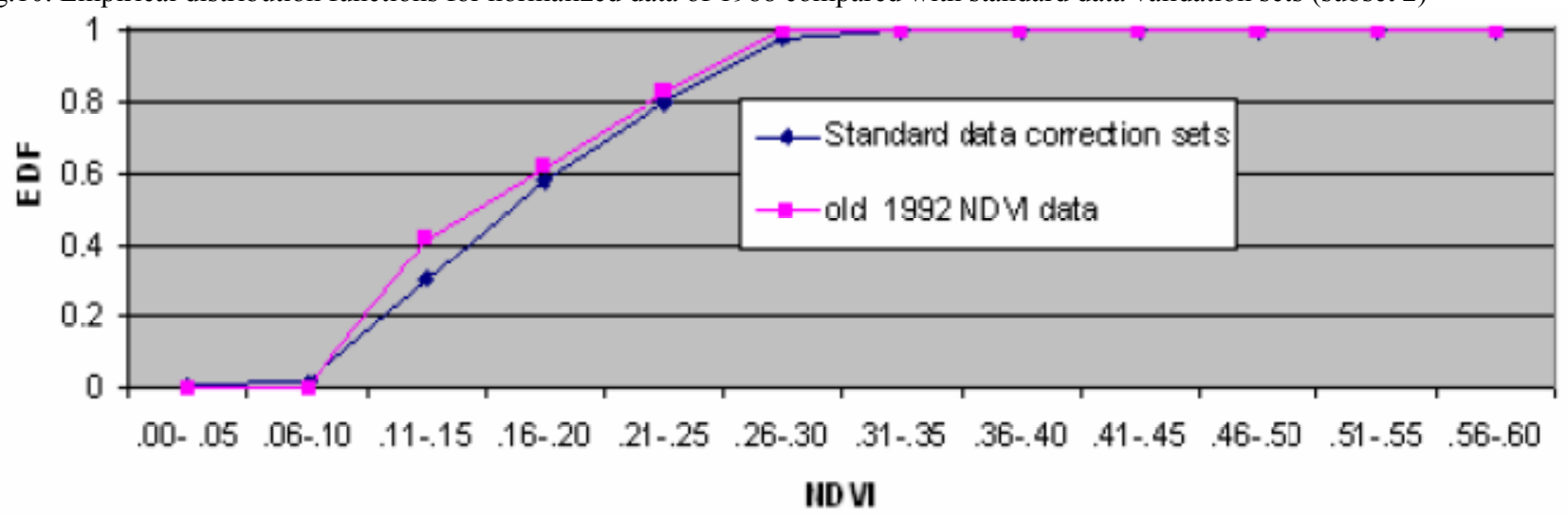

Fig.11. Empirical distribution functions for unnormalized data of 1992 compared with standard data correction sets (subset1) 


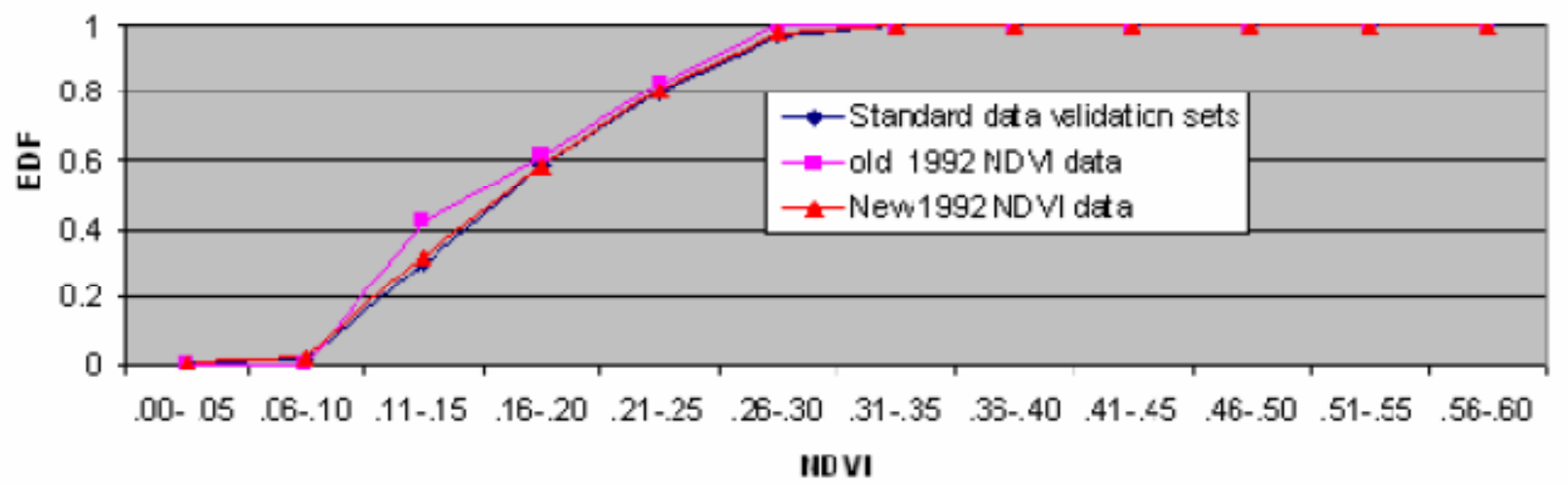

Fig.12. Empirical distribution functions for normalized data of 1992 compared with standard data validation sets (subset 2)

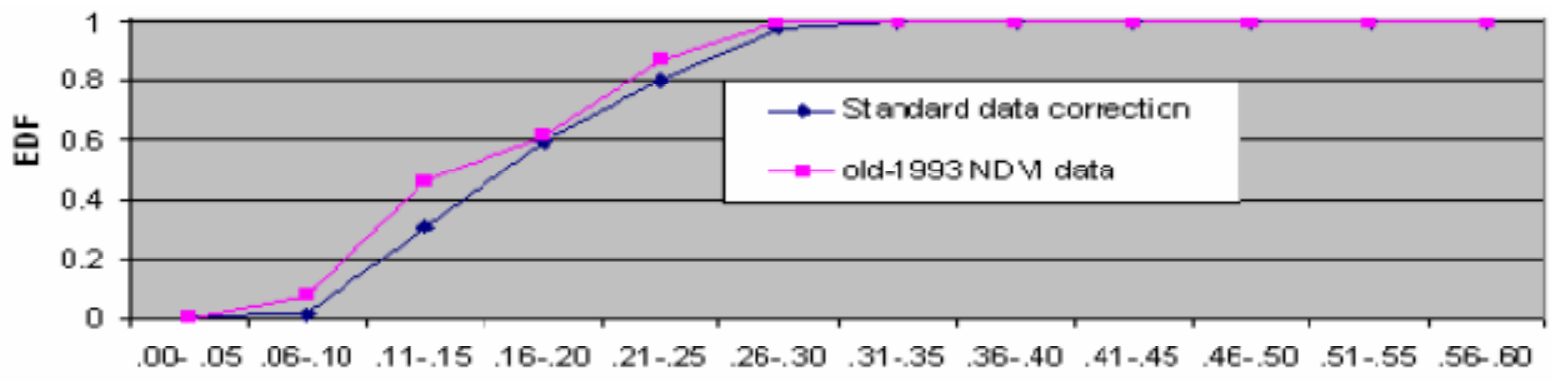

IID VI

Fig.13. Empirical distribution functions for unnormalized data of 1993 compared with standard data correction sets (subset1)

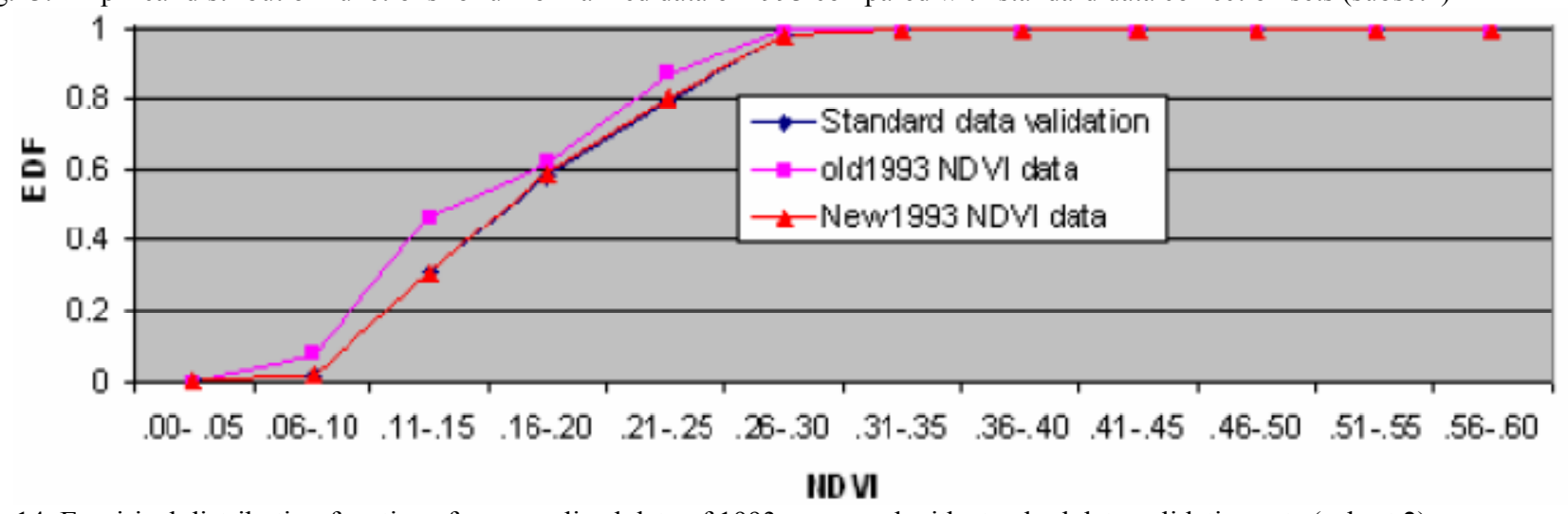

Fig. 14. Empirical distribution functions for normalized data of 1993 compared with standard data validation sets (subset 2)

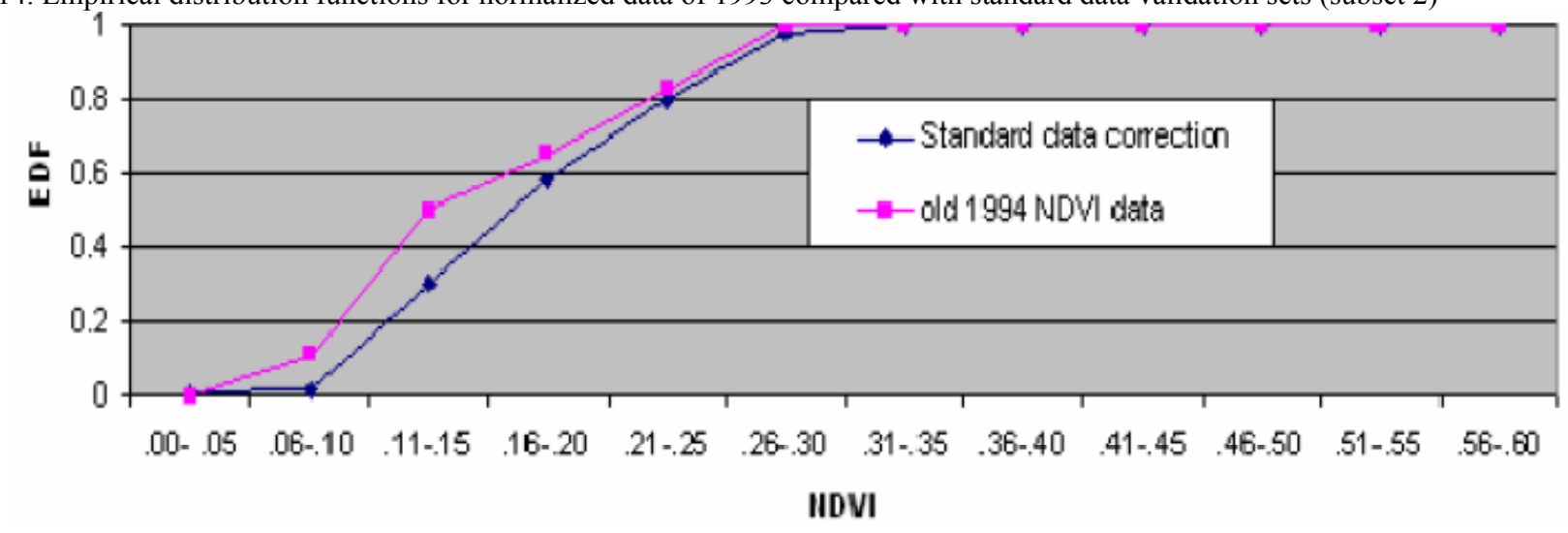

Fig.15. Empirical distribution functions for unnormalized data of 1994 compared with standard data correction sets (subset1) 


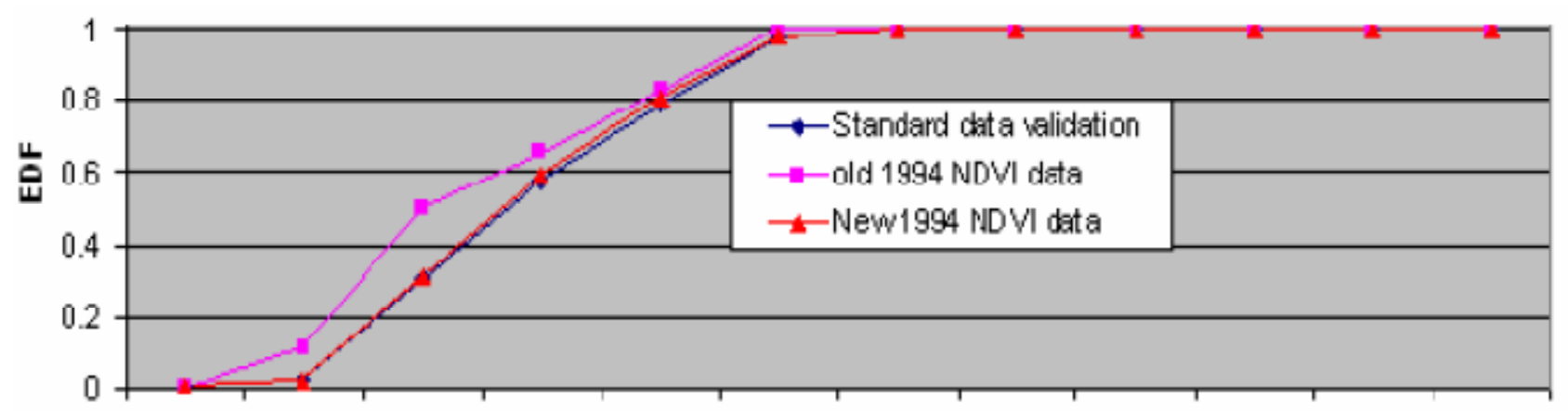

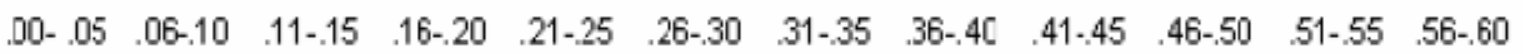

IID VI

Fig.16. Empirical distribution functions for normalized data of 1994 compared with standard data validation sets (subset 2)

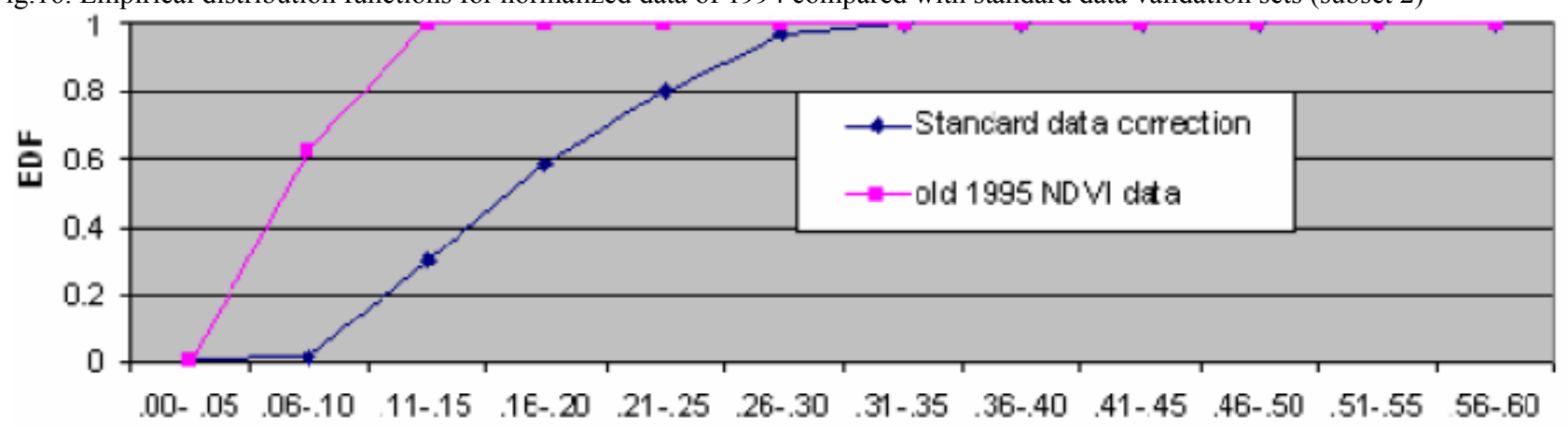

IIDVI

Fig.17. Empirical distribution functions for unnormalized data of 1995 compared with standard data correction sets (subset1)

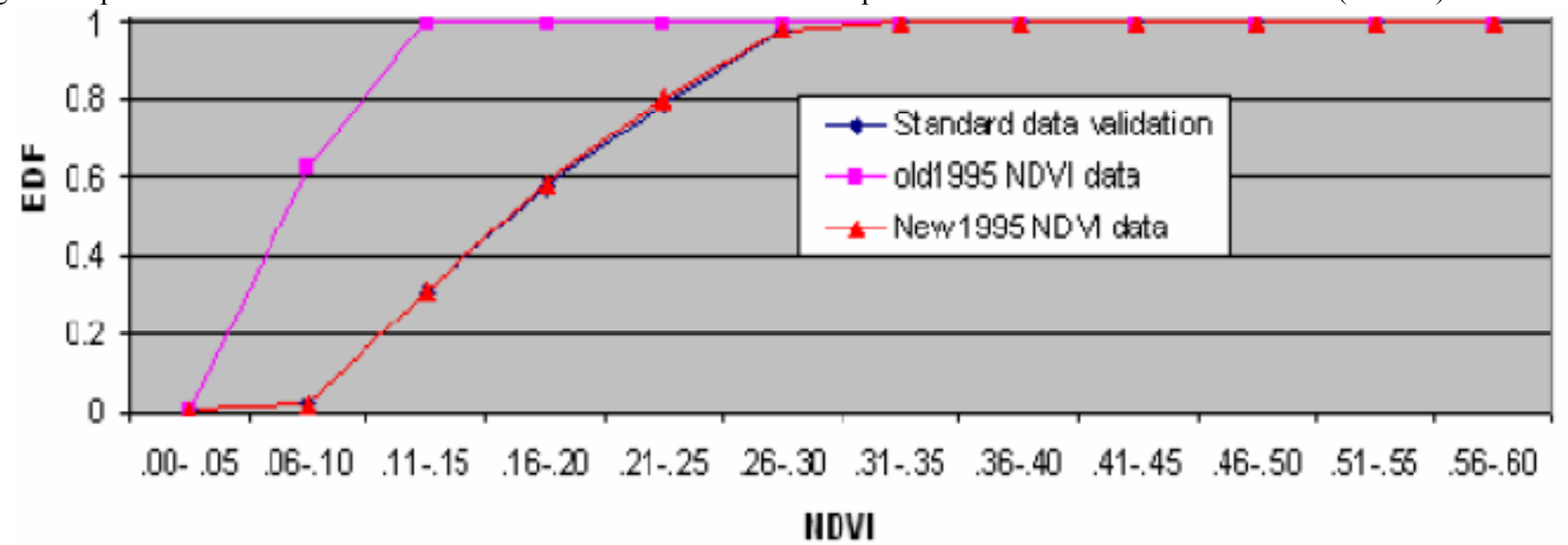

Fig.18. Empirical distribution functions for normalized data of 1995 compared with standard data validation sets (subset 2) 


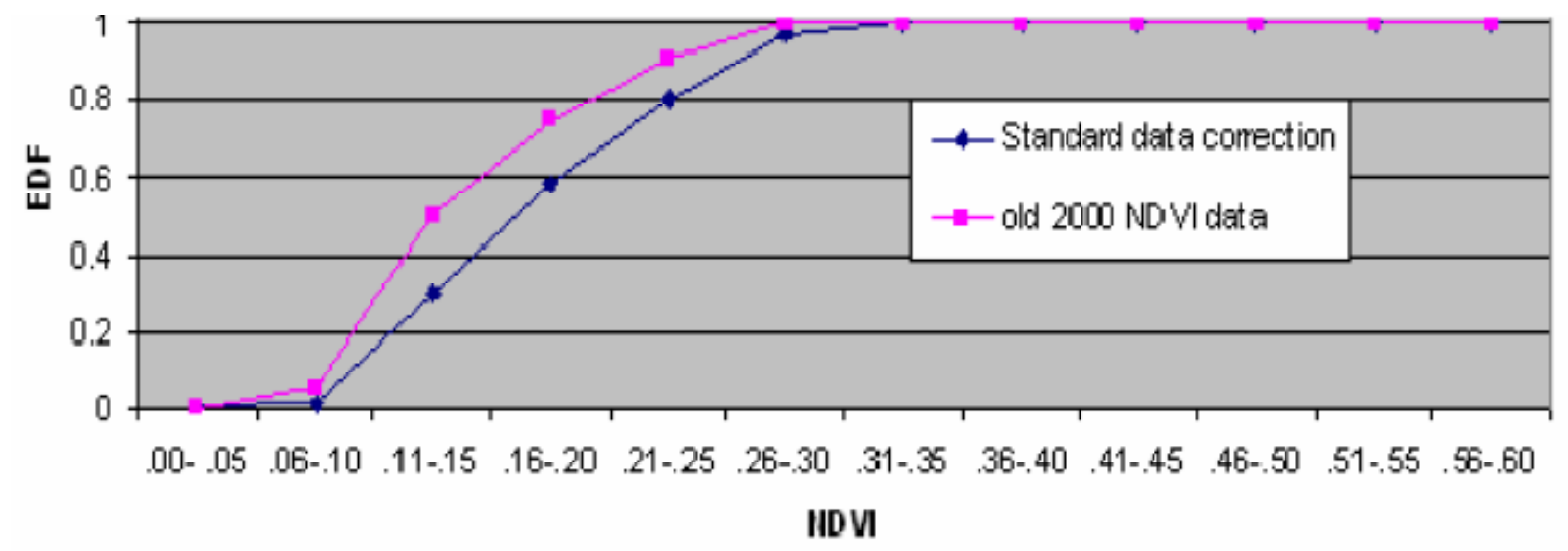

Fig.19. Empirical distribution functions for unnormalized data of 2000 compared with standard data correction sets (subset1)

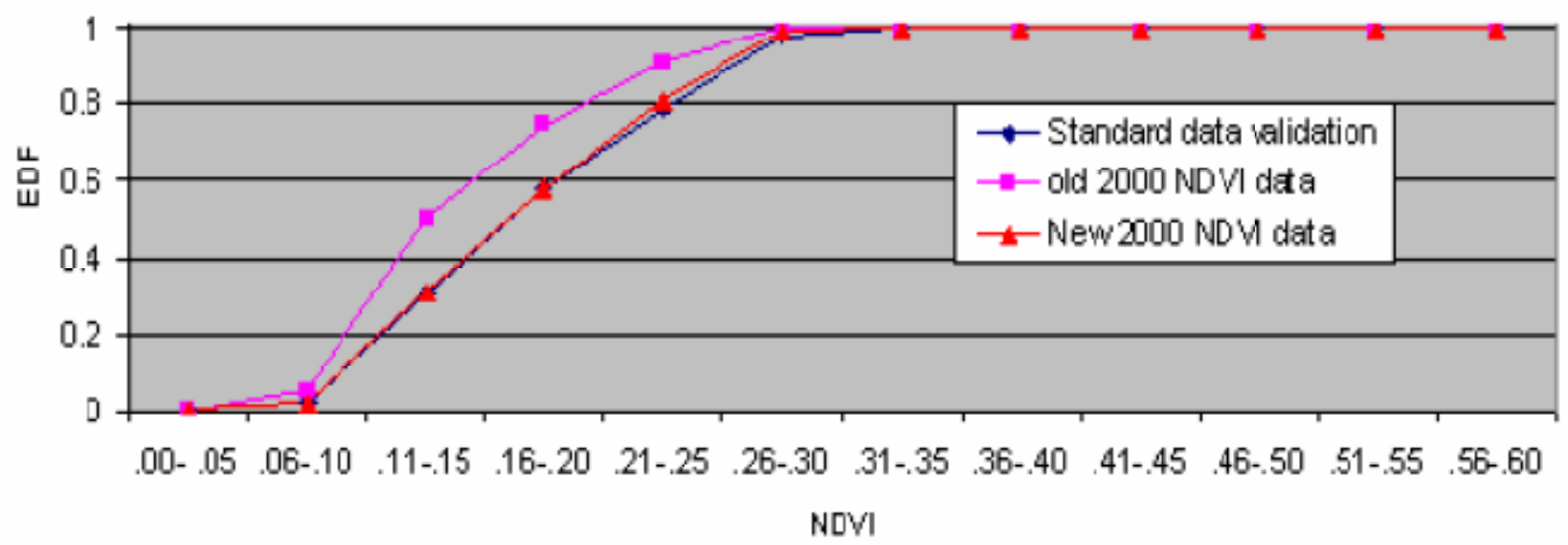

Fig.20. Empirical distribution functions for normalized data of 2000 compared with standard data validation sets (subset 2)

Fig.9-20 shows the EDF's of the normalize data for each of those years and indicates that the normalization was successful in making the EDF's of the two years nearly identical. This implies that the relationships between the EDF's remained essentially the same between two years. Those relationships, in fact, depend only on the relative function between two years. As long as the relative functions remain in the same, the normalization data remain effective. Using normalized value, we produce new NDVI time series for study area in China as shown in Figure 21 which shows improve the NDVI data (pink line) of the year of 1988, 1992, 1993, 1994, 1995, and 2000.

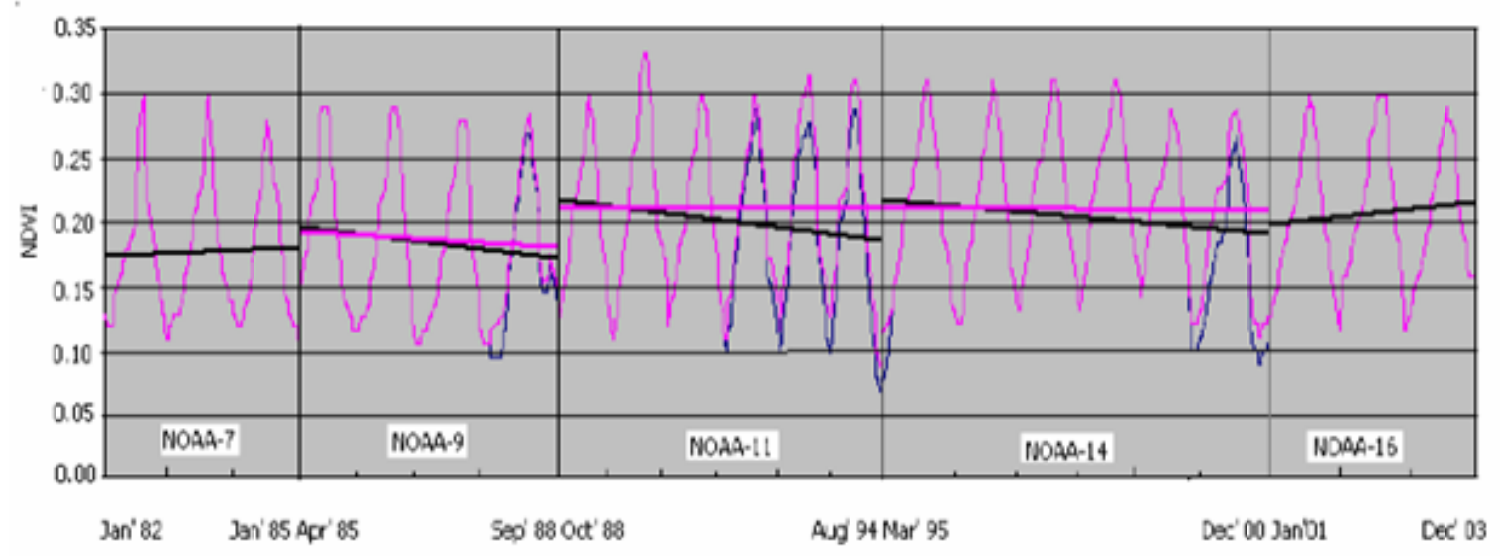

Fig. 21. New NDVI time series (yearly) for study area in China (old NDVI data - , and new NDVI data - ). 
NDVI trends for china and jumps between the satellites are illustrated in Figure 21 and the errors are estimated in Table 1. Figure 21 shows some NDVI trends for each satellite and jump from one satellite to the next one. Considering old NDVI trend (Table 1A), for china, NOAA-9, -11 , and -14 have negative trend and NOAA-7, -16 have positive trend. Therefore, NOAA-7, and -16 shows clear tendency to NDVI increase during its three years in operation. However, important is trend rate. Analysis shows that high rate of NDVI change for NOAA-9,-11, and -14 by reduction of NDVI in 1988, 1992-1994, and 2000 due to elevated amount of considerable degradation of satellite orbit.

Regarding NDVI jump from one satellite to the next in Table 1(B), general tendency is a reduction of NDVI between beginning of NOAA- 9 and the end of NOAA-7, between beginning of NOAA-16 and the end of NOAA-14. An increase in NDVI is observed only during satellite change from NOAA-9 to NOAA-11, NOAA-11 to NOAA-14, and NOAA-14 to NOAA-16 due to already mentioned orbit drift of satellite.

After correction of NDVI, we also estimate errors (new NDVI) in Table 1 of NDVI trends and jumps between the satellites. This table shows improve the NDVI trends for each satellite and jump from one satellite to the next one. The EDF method is designed to reduce only errors due to orbit drift, the dominant uncertainty in temperature variation during the satellite life time [6]. However, it may be difficult to accurately and completely remove this effect and thus orbit remains as an error source, though at a reduced level. Another large uncertainty lies in NDVI calibration and sensor degradation which includes all errors such as incomplete atmospheric corrections, surface corrections, sensor degradation and volcanic eruptions.

Table 1: Estimation of Errors in (A) NDVI trend at the End of a Satellite Life and (B) Jumps between the Satellites ( $\%$ to the beginning level)

\begin{tabular}{|c|c|c|c|c|c|c|c|c|c|c|}
\hline \multirow{2}{*}{\multicolumn{2}{|c|}{ Target }} & \multicolumn{5}{|c|}{ A } & \multicolumn{4}{|l|}{ B } \\
\hline & & N-7 & N-9 & $\mathrm{N}-11$ & $\mathrm{~N}-14$ & $\mathrm{~N}-16$ & $\mathrm{~N}-7 / 9$ & N-9/11 & $\mathrm{N}-11 / 14$ & $\mathrm{~N}-14 / 16$ \\
\hline \multirow[t]{2}{*}{ China } & $\begin{array}{l}\text { Old } \\
\text { NDVI }\end{array}$ & 3 & -10 & -12 & -11 & 7 & 10 & 30 & 16 & 5 \\
\hline & $\begin{array}{l}\text { New } \\
\text { NDVI }\end{array}$ & 3 & -5 & 0 & 0 & 7 & 7 & 19 & 0 & -5 \\
\hline
\end{tabular}

\section{CONCLUSIONS}

In this paper, the behavior of 22-year NOAA/NESDIS Global Vegetation Index (GVI) data is analyzed for improving the stability of the NDVI. Degradation of NDVI over time and shifts of NDVI between the satellites was estimated China data set, for it includes a wide variety or different ecosystems represented globally. Data for the years 1988, 1992, 1993, 1994, 1995 (first eight weeks), and 2000 are not stable enough compared to other years due to satellite orbit drift, And AVHRR sensor degradation, The orbit drift of the NOAA series satellites, among other factors, has a significant effect on the magnitude of the energy signal measured by the satellite sensors, and consequently on the values of NDVI .

To correct this error, we consider two techniques: empirical distribution functions, trend estimation method. The analysis of available techniques compared to other of the most optimum EDF. EDF is a statistical technique which allows to represent any global ecosystem from desert to tropical forest and to correct deviations in satellite data due to satellite technical problems (orbital drift, and AVHRR sensor degradation.). It is the best available technique to normalize satellite data. Empirical distribution function improves the time related stability of NDVI for all satellites, especially NOAA-9, -11, and -14 environmental satellites. Error exists when EDF's of unnormalized year and the standard data validation years are not identical. As a quantitative measure of the error, we express the differences in counts or percent, between the EDF of the standard and unnormalized years. Severity of the error was determined 
qualitatively from the AVHRR data and quantitatively with numerical measures of errors-- the empirical distribution functions themselves and count and percent difference among them. This is strong evidence that normalization by EDF matching is an effective method for improving stability of NDVI time series.EDF approach proposed here shows encouraging results which can be used globally to create vegetation index to improve the climatology.

\section{REFERENCES}

[1] Kogan, F.N., "Global draught watch from space," Bull. Am. Meteorol. Soc., 78, page 621- 636, (1997)

[2] Kogan, F.N., and X. Zhu, "Evolution of long-term errors in NDVI time series," Adv. Space Res. Vol. 28 (1), page 149-153(2001).

[3] Kogan, F.N., "Draught of the late 1980s in the United States as derived from NOAA polar orbiting satellite data," Bull. Amer. Metor. Soc. 76, page 655-668 (1995)

[4] Horn, B.K. P. and Woodham, R.J., "Destriping LANDSAT MSS images by histogram modification," Computer Graphics and Image processing. 10, page 69-83(1979)

[5] Gutman, G. Garik, "On the use of long-term global data of land reflactances and vegetation indices derived from the AVHRR," J. Geophys. Res., 104, 6, 6241-6255, (1999).

[6] Price, J. C., "Timing of NOAA afternoon passes,", Int. J. Remote Sens., 12, 193-198,(1991)

[7] Kidwell, K., "Global Vegetation User's Guide, NOAA," Natl. Environ. Satellite Data and Inf. Serv. Natl. Clim. Data Cent., Dep. Of Commer., Washington, D. C., 1997.

[8] Kidwell, K. B., NOAA Polar Orbiter Data Users Guide. U.S. Department of Commerce. National Oceanic and Atmospheric Administration, Satellite Data Services Division, Washington, D.C., 1991.

[9] P. Cracknell, The Advanced Very High Resolution Radiometer, Taylor \& Francis, page 90-97, (1997)

[10] Weinreb, M.P, RXie, J.H.Lienesch, D.S Crosby . "Removing stripes in GOES images by Matching Empirical Distribution Functions," NOAA Technical Memorandum NESDIS 26, May 1989

[11] Rao, C.R.N. and J.Chen, "Nonlinearity corrections for the thermal infrared channels of the Advanced Very High Resolution Radiometer": assessment and recommendations. NOAA Technical Report NESDIS 69 (Washington DC: US Department of Commerce, 1993a

[12] Rao, C.R.N. , "Degradation of the visible and near infrared channels of the Advanced Very High Resolution Radiometer on the NOAA -9 spacecraft," assessment and recommendations for corrections. NOAA Technical Report NESDIS 70 (Washington DC: US Department of Commerce). 1993b

[13] Rao, C.R.N. and J.Chen, "Post-launch calibration of the visible and near-infrared channels of the Advanced Very High Resolution Radiometer on NOAA-7, -9, and -11 spacecraft". NOAA Technical Report NESDIS 78 (Washington, DC: US Department of Commerce). 1994

[14] Rao, C.R.N. and J.Chen, "Postlaunch calibration of the visible and near-infrared channels of the advanced very high resolution radiometer on the NOAA 14 spacecraft", 1, Int. J. Remote Sens., 7, 2743-2747, (1996).

[15] Brest, C. L., and W. B. Rossow, "Radiometric calibration and monitoring of NOAA AVHRR data for ISCCP," Int. J. Remote Sens., 13, 235-273, (1992) 